\title{
Erratum: Gravitational Smoothing of Kinks on Cosmic String Loops [Phys. Rev. Lett. 118, 051301 (2017)]
}

\author{
Jeremy M. Wachter ${ }^{*}$ and Ken D. Olum \\ (Q) (Received 31 August 2018; published 3 October 2018)
}

DOI: 10.1103/PhysRevLett.121.149901

In the third paragraph following Eq. (4), we claim that kinks are not rounded off, because the force does not diverge due to $\Gamma_{\alpha \beta}^{\lambda}$ being bounded. This is incorrect. Instead, on one side of the kink, $\Gamma_{\alpha \beta}^{\lambda}$ grows as $\epsilon^{-1 / 3}$, where $\epsilon$ is the distance between the observation point and the kink. This divergence arises from the term in the Green's function inversely proportional to the distance between the observation point and the kink, which goes as $\epsilon^{1 / 3}$, combined with the discontinuous change in the source as one crosses the kink. A detailed analysis is in Ref. [1].

Because of this error, the claim that kinks are not rounded off is incorrect in general. However, in the specific cases of the Allen-Casper-Ottewill and Garfinkle-Vachaspati loops, the $\epsilon^{1 / 3}$ divergence is absent. Furthermore, because of the smallness of the $1 / 3$ power, the rounding off due to this effect moves outward slowly from the kink. The string direction near the kink location still changes rapidly, meaning that cusps produced by rounding off will initially be quite weak. Meanwhile, straightening out can still affect the string at any distance from the kink and on either side. We do not know which effect is more important in general.

*Department of Theoretical Physics, UPV/EHU, 48080 Bilbao, Spain. jeremy.wachter@ehu.es

[1] J. J. Blanco-Pillado, K. D. Olum, and J. M. Wachter, arXiv:1808.08254. 